\title{
Dynamic comparison of gut microbiota of mice infected with Shigella flexneri via two different infective routes
}

\author{
JINSONG YANG $^{1}$, WEI CHEN ${ }^{2}$, PINCHANG XIA ${ }^{2}$ and WENCHANG ZHANG ${ }^{1}$ \\ ${ }^{1}$ Fujian Provincial Key Laboratory of Environment Factors and Cancer, Key Laboratory of \\ Environment and Health, School of Public Health, Fujian Medical University, Fuzhou, Fujian 350122; \\ ${ }^{2}$ Department for Viral Disease Control and Prevention, Fujian Center for Disease \\ Control and Prevention, Fuzhou, Fujian 350001, P.R. China
}

Received May 29, 2019; Accepted December 6, 2019

DOI: $10.3892 /$ etm.2020.8469

\begin{abstract}
Shigella is one of the main pathogens causing diarrheal disease, and is associated with high morbidity and mortality in developing countries. Previous clinical data and animal studies have shown that the outcomes of oral and peritoneal infections of Shigella differ, and that the latter is more serious. Furthermore, a variety of pathogenic bacteria are known to cause changes in intestinal flora after infection, and the influence of Shigella infection on intestinal flora remains poorly understood. In the present study, the 16S rRNA high-throughput sequencing method was used to compare the changes in gut microbiota profiles in feces of mice infected with Shigella via two routes. In addition, the present study investigated the association between the differences in infection performance and bacterial communities. The present results suggested that the intraperitoneal route induced a distinct decrease in $\alpha$-diversity in the fecal microbiota when compared to the control at a later time, while the effect of the oral route on $\alpha$-diversity was not obvious. Oral infection of Shigella had a rapid and significant effect on gut microbiota, mainly causing a decreased abundance of Lactobacillus and an increased abundance of Prevotella and Escherichia/Shigella in the early stage of infection. By contrast, the effect of intraperitoneal infection on the gut microbiota was relatively slow and small. The principal coordinate analysis results suggested that the dynamic profile of gut microbiota between the two infective routes was consistent with the infection process. Probiotics, such as Lactobacillus reuteri and Faecalitalea exhibited significantly reduced abundance after Shigella infection. Collectively, the present results suggested that gut microbiota
\end{abstract}

Correspondence to: Professor Wenchang Zhang, Fujian Provincial Key Laboratory of Environment Factors and Cancer, Key Laboratory of Environment and Health, School of Public Health, Fujian Medical University, 1 Xue Yuan Road, Fuzhou, Fujian 350122, P.R. China E-mail: wenchang58@126.com

Key words: Shigella flexneri, gut microbiota, intraperitoneal route, oral route may play a pivotal role in the pathogenesis of Shigella infection. Future studies should investigate the effect of Shigella infection on the interaction between pathogenic bacteria and intestinal flora. The present results suggested that the use of probiotics may facilitate the prevention and treatment of shigellosis.

\section{Introduction}

Shigella is a common cause of bacterial dysentery, and can be classified into four serogroups: $S$. dysenteriae, S. flexneri, S. boydii and S. sonnei (1). Among these groups, S. flexneri is the most dominant pathogen found in developing countries $(2,3)$. The low-infectivity inoculum facilitates person-to-person spread by fecal-oral contact, which is the predominant mode of transmission (1). The clinical symptoms of shigellosis mainly include fever, diarrhea, abdomen convulsions and purulent bloody stools (4). The lesions are mainly confined to the colon, and result in mucosal congestion, edema and inflammatory cell infiltration between the ulcers (5) Bacteremia with Shigella is rare, especially in adults (6). In addition to the common route of oral infection, Shigella can also cause damage to multiple organs via the route of extra-intestinal infection, such as kidney and spleen abscesses induced by bacteremia $(7,8)$, perihepatitis-salpingitis caused by unhygienic sexual practices, and vulvovaginitis induced by fecal contamination $(9,10)$. In particular, when Shigella infection is combined with intestinal perforation, appendicitis and peritonitis, clinical manifestations of abdominal infection, such as high fever and septicemia, may occur with a high mortality rate (11). It was reported that 13 out of 57 children with surgical complications of Shigellosis died, despite having received antimicrobial therapy (12). In addition to the clinical reports, animal experiments have shown that diarrhea symptoms disappear within $24 \mathrm{~h}$ after oral infection with Shigella, while persistent diarrhea symptoms and mortality occur after intraperitoneal injection of Shigella (13). Therefore, the clinical manifestations of parenteral Shigella infection are more serious compared with those of intestinal infection, and the pathogenic mechanisms underlying these differences require further investigation.

Due to its long co-evolution with the host species, the gut microbiota influences numerous aspects of host physiology, 
including digestion, metabolism, immunity and behavior (14). In addition, the gut microbiota plays crucial roles in resisting the invasion and clearance of pathogenic microorganisms (15). Previous studies have shown that infectious diarrhea, such as that caused by Salmonella, Escherichia coli, parasites and viruses, is usually accompanied by changes in the intestinal flora (16-19). However, it remains unclear whether the gut microbiota in mice can be influenced by $S$. flexneri infection. In order to investigate the changes in intestinal flora after Shigella infection, and to identify the different clinical manifestations after oral or peritoneal infection based on gut microbiota changes, mice were challenged through two infective routes in the present study. The diversity and abundance of intestinal flora between these two groups were compared at different infection stages using $16 \mathrm{~S}$ ribosomal RNA (rRNA) high-throughput sequencing.

The results from the present study provided an insight into Shigella infection-induced gut microbiome changes, and identified potential biomarkers for Shigella infection, thereby facilitating the development of therapeutics for the prevention and treatment of shigellosis.

\section{Materials and methods}

Animals. Specific pathogen-free BALB/c mice (female; $\mathrm{n}=30$; weight, 18-22 g; age 6-8 weeks) were purchased from the Shanghai Laboratory Animal Center CAS, and housed at $22 \pm 2^{\circ} \mathrm{C}$ with $12 \mathrm{~h}$ light/dark cycle under specific pathogen-free conditions in the experimental facility for $\geq 1$ week prior to use in the experiments. The mice received a standard batch of food and water ad libitum. The present study was approved by The Institutional Animal Care and The Ethic Committee of Fujian Medical University.

S. flexneri treatment. The standard strain of S. flexneri (cat. no. 12022; American Type Culture Collection) was inoculated in Gram Negative Enrichment Broth (HuanKai Microbial Sci. and Tech. Co., Ltd.) overnight at $37^{\circ} \mathrm{C}$ with shaking at $180 \mathrm{rpm}$ on an air table. After the culture reached the logarithmic growth stage (0.4-0.5 optical density at $600 \mathrm{~nm}$ ), the suspension was centrifuged at $825 \mathrm{x} \mathrm{g}$ for $1 \mathrm{~min}$ at $4^{\circ} \mathrm{C}$ to collect the bacterial precipitate. Then, this was suspended in normal saline buffer. The mice were randomly divided into three groups ( $n=10$ in each group): i) Control group with normal saline gavage; ii) intraperitoneal (IP) injection group, $5 \times 10^{7}$ colony-forming units (CFU) of Shigella given by IP injection; and iii) oral gavage group, $5 \times 10^{8} \mathrm{CFU}$ of Shigella given orally. Each mouse was administered the treatment at a volume of $0.1 \mathrm{ml}$. The administered bacterial doses were confirmed by plating serial dilutions onto Salmonella-Shigella agar plates (HuanKai Microbial Sci. and Tech. Co., Ltd.). After treatment with S. flexneri, the mice received sterilized food and water ad libitum for 7 days, and were then sacrificed. During this period, body weight, general physical activity, fur ruffling, abdominal swelling and body temperature of the mice in each group were recorded every day. To calculate the survival rates of mice infected with Shigella in the groups, Olfert's guidelines on humane endpoints (20) were adopted as follows: When a mouse lost $>20 \%$ of its body weight, showed signs of coma and lethargy, and had a body temperature $<34^{\circ} \mathrm{C}$, the mouse was considered to have reached a humane endpoint and was euthanized. The remaining mice were euthanized 7 days after Shigella infection.

Fecal sample collection. To evaluate $S$. flexneri shedding in the feces, one piece of fecal pellet was collected and weighed (0.1-0.3 g) from each mouse at the time points of $0,6,12$, 24 and $48 \mathrm{~h}$ after infection. Then, these were suspended in sterile PBS by extensive vortexing to prepare a uniform fecal suspension, which was cultivated on Salmonella-Shigella agar (HuanKai Microbial Sci. and Tech. Co., Ltd.) by incubation overnight at $37^{\circ} \mathrm{C}$ and the number of colonies was counted. For $16 \mathrm{~S}$ high-throughput sequencing, five fresh feces samples per day were randomly collected from five mice in each group using aseptic technique on days 2, 3 and 4 after the administration of Shigella. Three pieces of feces from each mouse were combined to be a single sample to meet the requirement of sample size. In total, 45 samples of feces were collected which were immediately frozen in liquid nitrogen and stored at $-80^{\circ} \mathrm{C}$ for further analysis.

DNA extraction, PCR amplification and $16 \mathrm{~S}$ rRNA sequencing. Bacterial genomic DNA was extracted from the stool samples using a QIAamp Fast DNA Stool Mini kit (cat. no. 51604; Qiagen $\mathrm{GmbH}$ ), according to manufacturer's protocol. The hypervariable (V)3-V4 region of the bacterial 16S rRNA was amplified by PCR (ABI GeneAmp ${ }^{\circledR} 9700$, USA) with Phusion ${ }^{\circledR}$ High-Fidelity DNA Polymerase (New England BioLabs, Inc.) under the following conditions: Initial denaturation at $95^{\circ} \mathrm{C}$ for $5 \mathrm{~min}$; followed by 30 cycles of $95^{\circ} \mathrm{C}$ for $30 \mathrm{sec}, 55^{\circ} \mathrm{C}$ for $30 \mathrm{sec}$ and $72^{\circ} \mathrm{C}$ for $30 \mathrm{sec}$; and a final extension at $72^{\circ} \mathrm{C}$ for $4 \mathrm{~min}$. The resulting PCR product was stored at $4^{\circ} \mathrm{C}$. The universal primers used in the PCR reaction were $341 \mathrm{~F}$ (5'-CCT AYGGGRBGCASCAG-3') and 806R (5'-GGACTACNNGGG TATCTAAT-3'). The 5'-end of 314F carried a special sequence tag to encode each sample. The obtained PCR products were separated by electrophoresis using $2 \%$ agarose gel, and purified using the GeneJET Gel Extraction kit (Thermo Fisher Scientific, Inc.). Then, DNA libraries were constructed using an Ion Plus Fragment Library kit (Thermo Fisher Scientific, Inc.) and sequenced using an Ion S5 XL system (Thermo Fisher Scientific, Inc.), according to manufacturer's protocol. Raw FASTQ files were pretreated and quality-filtered using the Quantitative Insights Into Microbial Ecology (QIIME) bioinformatics pipeline (version 1.17; http://qiime.org/). Next, the sample data were separated from the reads obtained according to the barcodes, and the barcodes and primer sequences were removed to obtain raw reads. After data filtering, the remaining high-quality clean data were assembled based on tags and sequence overlaps.

Data analysis. For the analysis of microbial diversity, operational taxonomic units (OTUs) were generated for all sequences based on different similarity levels (21). In the present study, the OTUs were clustered according to a similarity cutoff value of 97\% using UPARSE software (version 7.1; http://drive5. com/uparse/), while USEARCH (version 10.0.240, http://www. drive5.com/usearch/) was used to identify and remove chimeric sequences. SSUrRNA database of SILVA132 (http://www. 

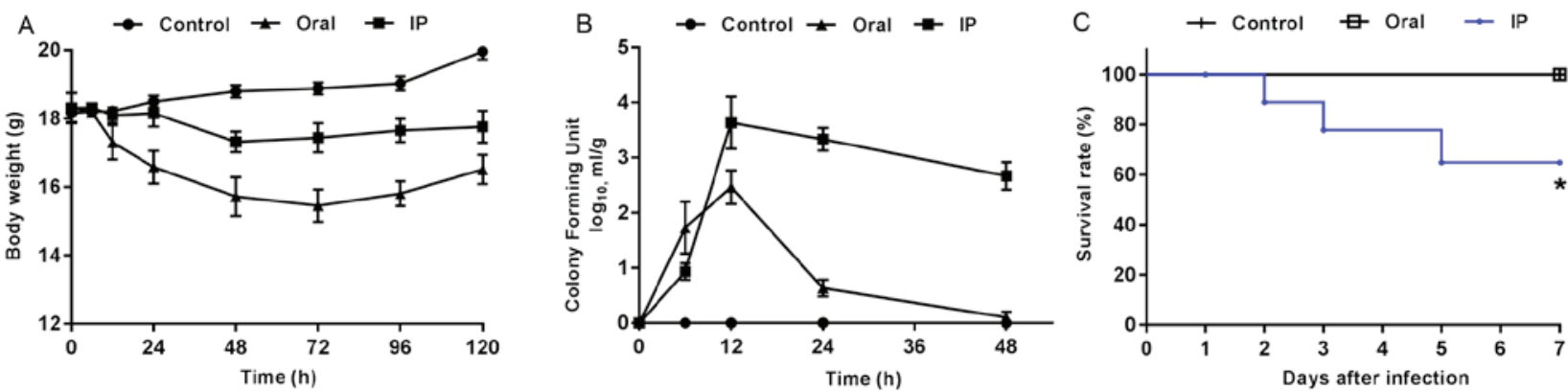

Figure 1. General status of mice after infection in the different groups. (A) Body weight changes were monitored at different post-infection time points. Data are presented as the mean \pm SEM. $n=10$ mice/group. (B) Colony-forming units in fecal homogenates are expressed as the mean \pm SEM from six mice/group at different time points. (C) Survival curves indicate the survival rates of mice in different groups. ${ }^{~} \mathrm{P}<0.05$ vs. control group. IP, intraperitoneal injection group.

arb-silva.de) was selected for species annotation analysis, where the threshold was set as 0.8-1). The taxonomy of $16 \mathrm{~S}$ rRNA gene sequences was analyzed using the Ribosomal Database Project (RDP) classifier (version 2.11; http://sourceforge.net/projects/rdp-classifier/), and the sequences were classified to different levels, including phylum, class, order, family, genus and species. Subsequently, the composition and relative abundance of each sample at each classification level were calculated. The OTU profiling table and $\alpha$-diversity indices, including Chaol, Shannon and Simpson (22), were calculated using QIIME. The $\beta$-diversity among different groups was analyzed by principal coordinate analysis (PCoA) based on weighted UniFrac distances (23). Bray-Curtis dissimilarities were used to construct a hierarchical cluster, while non-metric multidimensional scaling (NMDS) was plotted based on the weighted UniFrac dissimilarity (24). Linear discriminant analysis Effect Size (LEfSe) was used to find the biomarkers with statistical differences between groups, which was presented by Histogram of Linear Discriminant Analysis distribution and cladogram (25). Data are presented as the mean \pm SEM. The statistical analyses were performed with a one-way ANOVA followed by Tukey's post-hoc test, while Kruskal-Wallis analysis followed by Dunn's multiple comparisons test was used for data with variance inhomogeneity using SPSS 19.0 (IBM Corp.). $\mathrm{P}<0.05$ was considered to indicate a statistically significant difference. GraphPad Prism (version 7.0; GraphPad Software, Inc.) was used to compare mouse survival rates between the groups using a Mantel-Cox test, and to generate a graphical representation of the results.

\section{Results}

General condition of mice after infection. The present results suggested that the animals challenged with $S$. flexneri via IP and oral routes exhibited marked changes in physical activity, food intake, fur ruffling and abdominal swelling. At $2 \mathrm{~h}$ after the oral administration of $S$. flexneri, mice began to show diarrhea symptoms, which were evident at $6 \mathrm{~h}$ and gradually disappeared after $24 \mathrm{~h}$. At $12 \mathrm{~h}$ after IP injection, mice began to present with diarrhea, some of which lasted until day 7. Furthermore, pronounced abdominal edema was observed, and mice presented with high irritability upon touching of the abdomen. Compared with the control group, all mice in the infection groups presented with varying degrees of weight loss within 3 days after $S$. flexneri infection (Fig. 1A). The mice infected orally with S. flexneri had greater weight loss compared with both the controls and the IP group. In addition, the CFU value of $S$. flexneri detected in the feces of the oral group peaked at $12 \mathrm{~h}$, and then exhibited a rapid decline (Fig. 1B). In the IP group, the maximum CFU value also appeared at $12 \mathrm{~h}$, but was followed by a slower rate of decline compared with that in the oral group. During the experiment, three mice in the IP group reached the humane endpoint which were euthanized and excluded from the further experiments, while none of the mice in the control or oral groups died. The present results suggested that the differences between the survival rates in the three groups was statistically significant (Fig. 1C; $\mathrm{P}=0.019$ ).

$\alpha$-diversity. In the present study, 3,517,768 high-quality reads were generated from the 45 samples by filtering the chimera. In total, $\sim 78,172$ reads were obtained from each sample for further analysis, and the average read length was $415 \mathrm{bp}$. Then, OTU clustering was performed with $97 \%$ identity and 1,468 OTUs were obtained by removing those with low abundance (threshold, $0.005 \%$ ). These were then annotated with the species from the Silva132 database. The present results suggested that a total of $738(50.27 \%)$ OTU annotations were made at the genus level. Furthermore, there were significant differences in $\alpha$-diversity between the IP and control groups on day $4(\mathrm{P}<0.05$ and $\mathrm{P}<0.01$; Table $\mathrm{I})$. The Shannon index and Chaol were lower in the IP group on day 4 compared with the control group, while there were no significant differences between the oral group and control group.

Taxonomic differences between groups at different time points. The relative abundances of OTUs from different groups at different time points were compared, and the relative abundances of the top ten bacterial species at the level of phylum and family are presented in Fig. 2. The present results suggested that almost all OTUs belonged to the following five phyla: Bacteroidetes, Firmicutes, Proteobacteria, Deferribacteres and unidentified_Bacteria, accounting for $>99 \%$ of the total OTUs. The main taxa at the phylum level, family level and genus level at different time points (days 2, 3 and 4) in the three groups were compared (Fig. 3). At the phylum level, the oral group showed a higher relative abundance of Bacteroidetes and Proteobacteria on day 2 compared with the control group and IP group $(\mathrm{P}<0.05)$, but had lower abundance of Firmicutes compared with the control group 
Table I. $\alpha$-diversity indices of the gut microbiota from the three groups at different time points.

\begin{tabular}{lcccccc}
\hline Time & Group & $\begin{array}{c}\text { Average length } \\
\text { of sequence, bp }\end{array}$ & Effective reads & Shannon index & Simpson index & Chao1 \\
\hline Day 2 & C & 415 & 80140 & $6.74 \pm 0.58$ & $0.97 \pm 0.02$ & $575.21 \pm 36.30$ \\
& IP & 413 & 80133 & $6.51 \pm 0.35$ & $0.98 \pm 0.01$ & $552.17 \pm 20.87$ \\
& Oral & 415 & 80146 & $6.71 \pm 0.20$ & $0.98 \pm 0.01$ & $545.29 \pm 35.02$ \\
Day 3 & C & 417 & 82278 & $6.64 \pm 0.21$ & $0.97 \pm 0.01$ & $574.90 \pm 116.48$ \\
& IP & 418 & 80872 & $5.96 \pm 0.41$ & $0.98 \pm 0.01$ & $517.84 \pm 127.05$ \\
Day 4 & Oral & 418 & 72138 & $6.65 \pm 0.51$ & $0.97 \pm 0.01$ & $532.22 \pm 155.22$ \\
& C & 415 & 80225 & $6.73 \pm 0.31$ & $0.97 \pm 0.01$ & $600.88 \pm 96.70$ \\
& IP & 415 & 80170 & $6.02 \pm 0.21^{\mathrm{a}}$ & $0.97 \pm 0.01$ & $424.79 \pm 29.74^{\mathrm{b}}$ \\
& Oral & 413 & 80151 & $6.49 \pm 0.33$ & $0.97 \pm 0.01$ & $634.16 \pm 82.14$ \\
\hline
\end{tabular}

${ }^{\mathrm{a}} \mathrm{P}<0.05,{ }^{\mathrm{b}} \mathrm{P}<0.01$ vs. control group. IP, intraperitoneal; C, control.
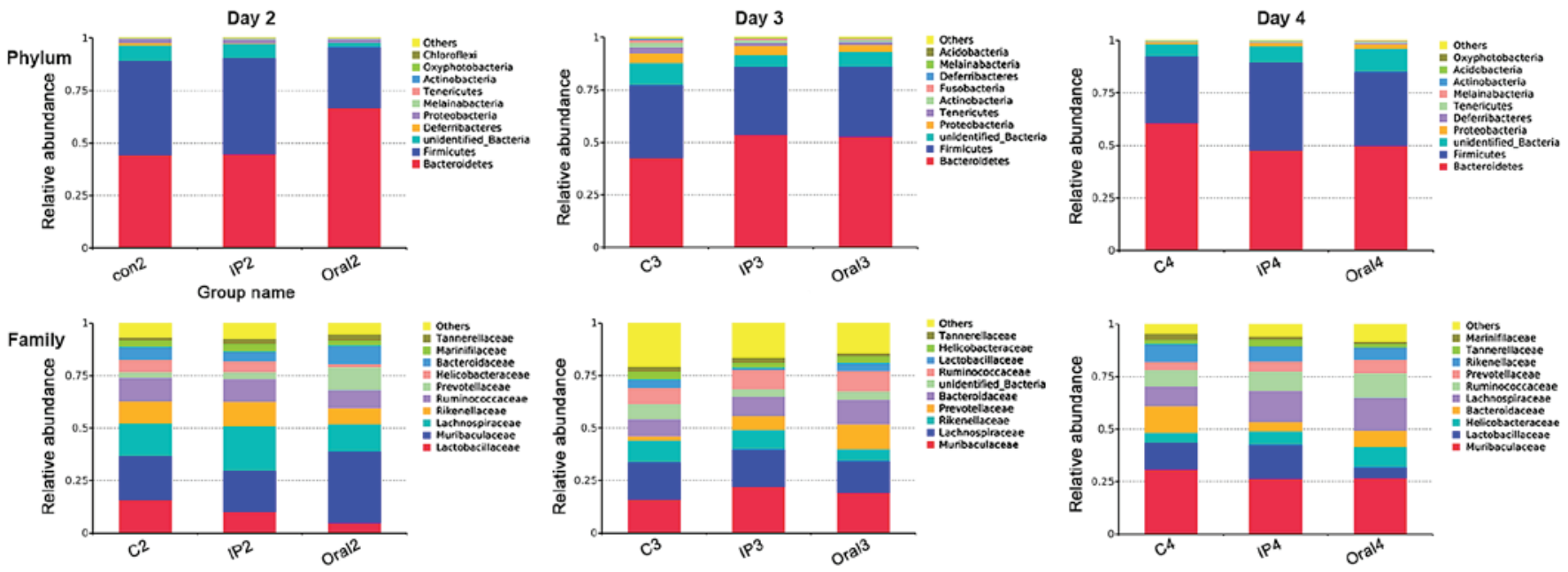

Figure 2. Relative percentage abundance of the main taxa at the phylum level and family level in the three groups. Relative percentage abundance was assessed using 16S high-throughput sequencing at days 2, 3 and 4 . The sequences that could not be classified into any known group were designated as 'unidentified_Bacteria'. C, control; IP intraperitoneal.

$(\mathrm{P}<0.05)$. The present results suggested that on day 4 , the relative abundance of Melainabacteria in the oral group was significantly higher compared with the control group and IP group $(\mathrm{P}<0.05)$. At the family level, on days 2 and 3 the relative abundance of Lactobacillaceae in the oral group was significantly lower compared with the control group $(\mathrm{P}<0.01$ and $\mathrm{P}<0.05$, respectively); while it was lower in the IP group compared with the control group, the difference was not statistically significant. On days 2 and 3, the relative abundance of Prevotellaceae bacteria in the oral group was higher compared with the control group $(\mathrm{P}<0.05)$. The relative abundance of Muribaculaceae, belonging to the phylum Bacteroidetes, on day 2 in the oral group was significantly higher compared with the control group and IP group $(\mathrm{P}<0.05)$. In addition, the present results suggested that the relative abundance of Lachnospiraceae on day 4 in the oral group was significantly higher compared with the control group. At the genus level, the relative abundances of Lactobacillus and Alistipes in the oral group on days 2 and 3 were significantly lower compared with the control group $(\mathrm{P}<0.05$ and $\mathrm{P}<0.01)$. By contrast, the relative abundance of Alloprevotella was significantly higher in the oral group compared with the control group and IP group $(\mathrm{P}<0.05$ and $\mathrm{P}<0.01)$. The proportion of Escherichia-Shigella was significantly higher in the IP group and oral group on days 2, 3 and 4 compared with the control group $(\mathrm{P}<0.05$ and $\mathrm{P}<0.01)$.

$\beta$-diversity. $\beta$-diversity describes the microbial community composition and can be used to evaluate the differences among microbial communities (22). In the present study, PCoA analysis was performed based on the weighted UniFrac distance, and then the main coordinate combination with the highest contribution rate was selected for plotting. The present results suggested that there were different distribution styles between the IP and oral groups at each sampling point (days 2, 3 and 4). On day 2, PC1 represented $67.69 \%$ of the variation between all the groups, and the main coordinate analysis could distinguish the oral group from the other two groups (Fig. 4A). 
A
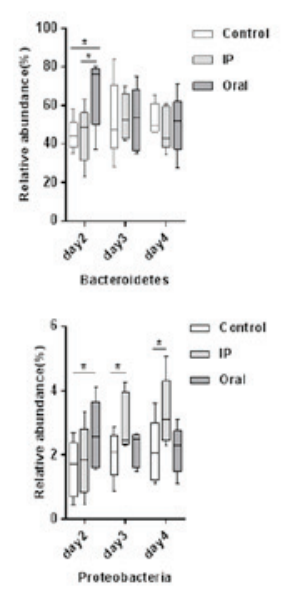

B
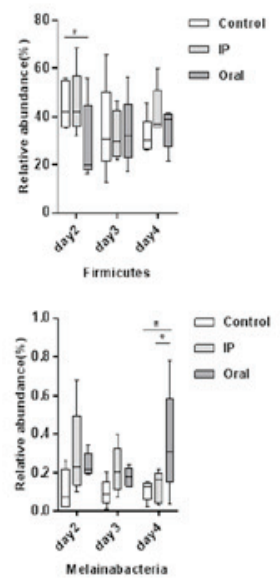
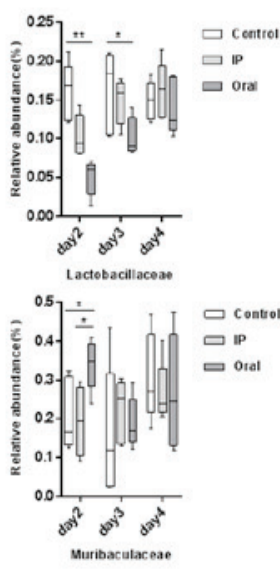

C
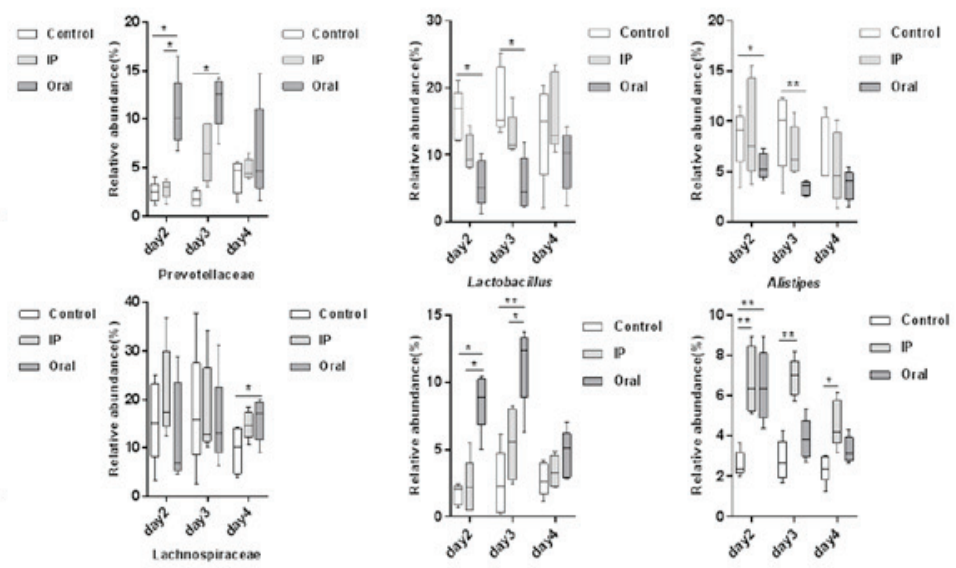
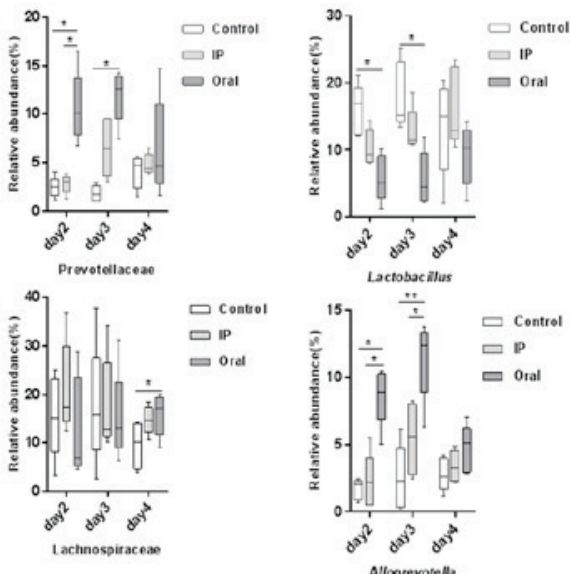

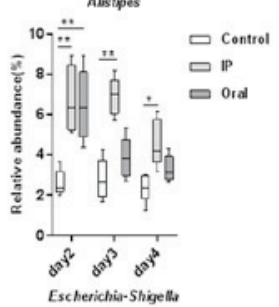

Figure 3. Comparison of the relative abundance in the main taxa among the three groups at different time points. Relative abundance at (A) the phylum level, (B) family level and (C) genus level. ${ }^{*} \mathrm{P}<0.05,{ }^{* *} \mathrm{P}<0.01$. IP, intraperitoneal.

A

Day 2

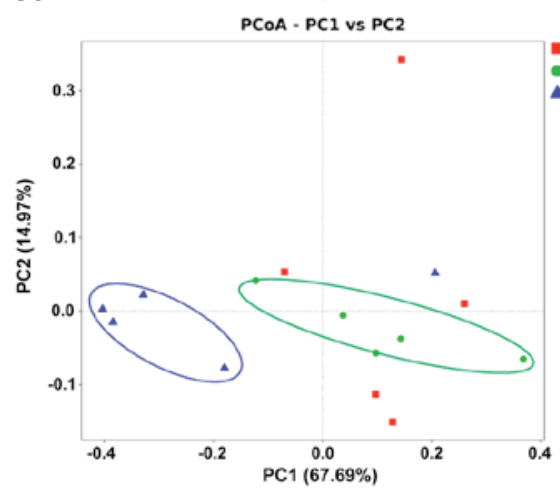

B

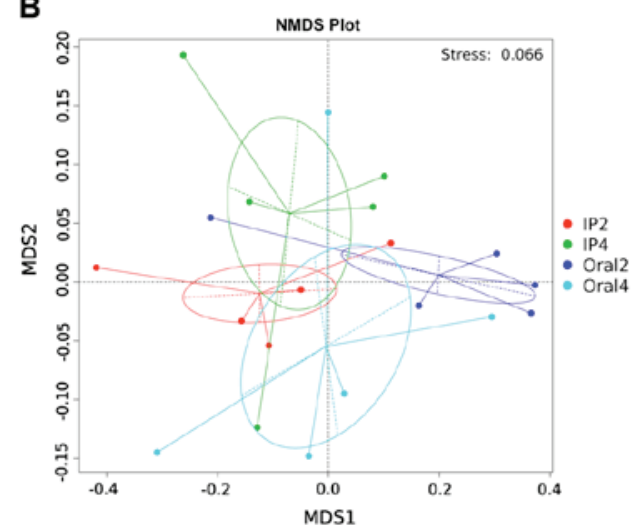

Day 3

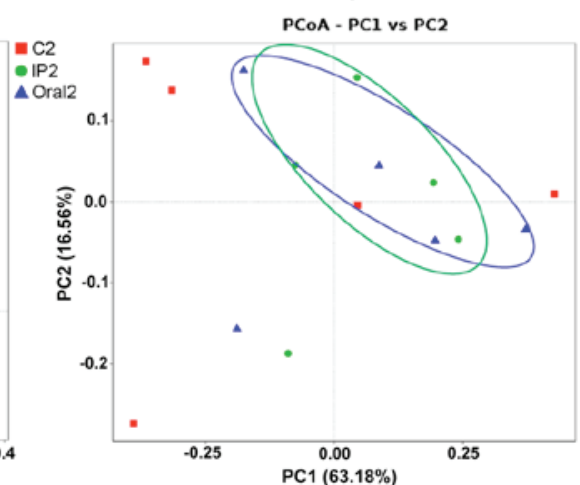

C
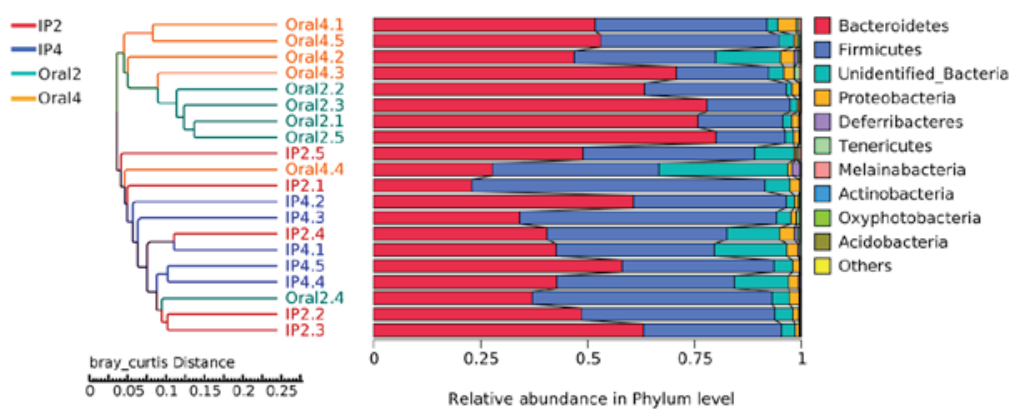

Day 4

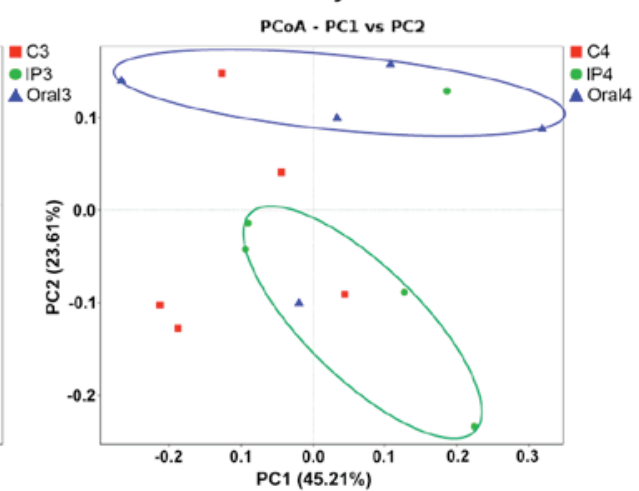

.

Figure 4. Analysis of the time-course of S. flexneri infection in mice. (A) PCoA of fecal microbiota on days 2, 3 and 4, based on the weighted UniFrac dissimilarity index. (B) Non-metric multidimensional scaling plot for the gut microbiota of the IP group and oral group on days 2 and 4, based on the weighted UniFrac dissimilarity index. (C) Hierarchical cluster analysis of operational taxonomic units in the IP group and oral group on day 2 and 4, based on the Bray-Curtis dissimilarity. IP, intraperitoneal; PCoA, principal coordinate analysis; NMDS, non-metric multidimensional scaling; MDS, metric multidimensional scaling.

Furthermore, the coordinates of the samples in the IP group and control group were similar. On day 3, the coordinates for the IP group overlapped with those of the oral group (Fig. 4A). On day 4, the positions of the IP group and the oral group were separated, and their coordinates were different compared with the control group, indicating a PC1 value of $45.17 \%$ between the three groups (Fig. 4A). NMD analysis showed the degree of isolation of intestinal flora between the groups at different time points. The present results identified varying degrees of location differences between the IP group and the oral group on days 2 and 4 (Fig. 4B). The hierarchical cluster analysis of OTUs indicated that the IP and oral groups were well-separated. The present results suggested that the samples in the oral group (Oral2 and Oral4) were clustered according 
A

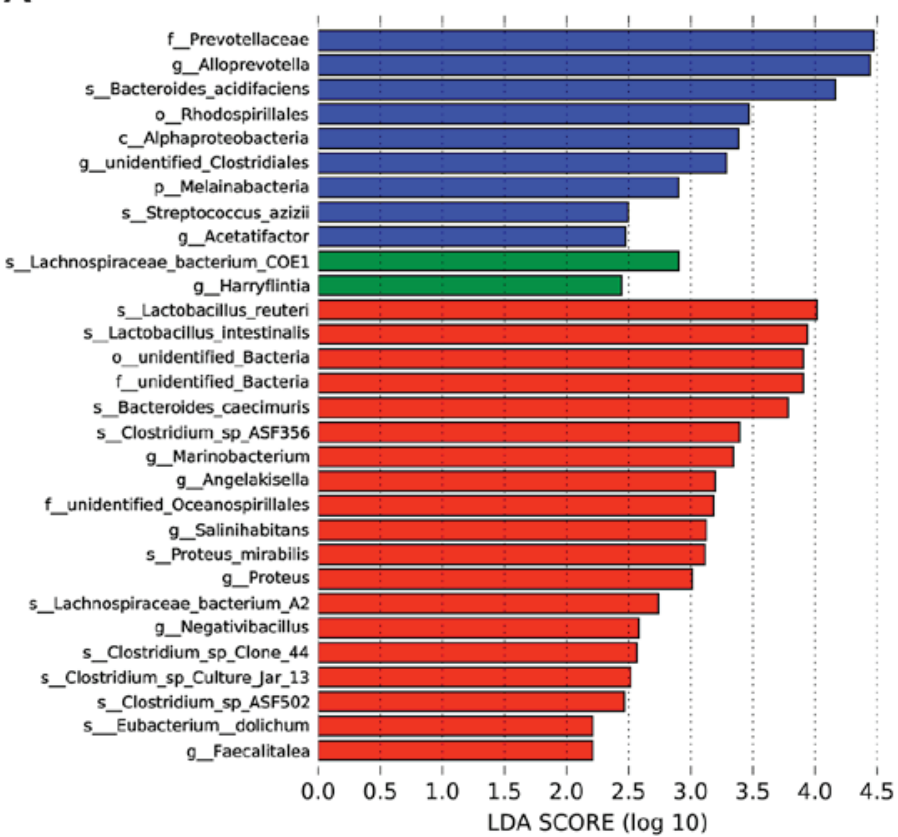

B

Cladogram

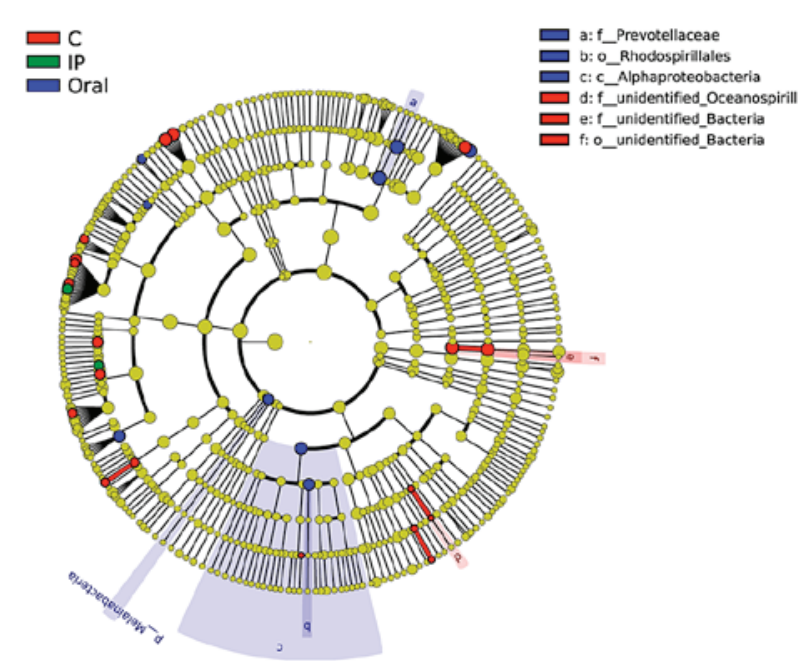

Figure 5. Microbial biomarkers among the different groups. (A) Linear discriminant analysis Effect Size analysis indicated the differentially abundant taxa as biomarkers. Kruskal-Wallis test produced $\mathrm{P}<0.05$ and an LDA score of $>2.0$. (B) The cladogram of taxa abundances in different groups. Taxa without significant differences are labeled in yellow, while significantly different taxa are labeled using the color of each group, red represents control, green represents the IP group and blue represented the oral group. C, control; IP, intraperitoneal; LDA, linear discriminant analysis.

to the time points, while samples in the IP group (IP2 and IP4) intersected the cluster tree to a certain extent (Fig. 4C).

LEfSe analysis. LEfSe, the computational approach to biomarker class comparisons contributes to the understanding of microbial communities (25). In order to assess the gut microbial responses associated with $S$. flexneri infection at the taxonomic level, microbial clade differences were determined using LEfSe analysis. The present results suggested that a total of 19 bacterial taxa, such as Lactobacillus reuteri, Lactobacillus intestinalis, Bacteroides caecimuris and Faecalitalea, had a higher abundance in the control group compared with the IP group and oral group (Fig. 5A). However, the relative abundance of nine taxa of bacteria, such as Prevotellaceae, Alloprevotella and Bacteroides acidifaciens, in the oral group was higher compared with the control group and IP group (Fig. 5A). In addition, only two taxa of bacteria in the IP group were in higher abundance compared with the other groups. The present results suggested that three dominant bacteria were found in the control group, while Prevotellaceae, Rhodospirillales and Alphaproteobacteria were dominant in the oral group (Fig. 5B).

\section{Discussion}

Infections with Shigella spp. are usually self-limiting and confined to the mucosa of the distal ileum and the colon (26). However, in rare cases, parenteral infection can lead to bacteremia, multiple organ infections and abscesses (7). In order to investigate the differences in the effects of Shigella intestinal infection and parenteral infection on gut microbiota, and the possible role of gut microbiota in the different infection routes, a Shigella infection mouse model was established by IP injection and gavage in the present study. The present results suggested that adult mice infected by oral infection and intraperitoneal injection exhibited different disease outcomes. Oral infection gradually self-cured within 3 days, while the IP infection developed into persistent diarrhea and other symptoms of dysentery. In the IP group, $S$. flexneri was constantly detected in the feces and a certain proportion of mice died, which is consistent with previous results from Yang et al (13) and Sharma et al (27) Furthermore, 4 day old mice (28) and young mice treated with antibiotics (29) are frequently used to construct animal models of shigellosis via oral administration. However, both these models neglect the role of the underdeveloped gut microbiome in the pathogenesis of shigellosis. By contrast, the adult mice included in the present study had a developed gut microbiome, which may better reflect the in vivo effects of Shigella on the gut microbiome.

$\alpha$-diversity indexes, including Chao1, Shannon, Simpson, Good's coverage and the Abundance-based Coverage Estimator, can be used to analyze the abundance and diversity of microbial communities (30). In the present study, no significant change in $\alpha$-diversity was identified after oral Shigella infection in mice. A possible explanation may be that Shigella was cleared in a relatively short time under the action of the intestinal immune system of mice (31); hence, the effect of Shigella on the intestinal flora was short-lived and limited. Only Bacteroidetes, Firmicutes and Proteobacteria with high relative abundance were significantly affected, while other bacteria with low abundance were less affected. Therefore, the present results suggested that there was no significant change in the $\alpha$-diversity of the gut microbiota after oral infection. In addition, the $\alpha$-diversity in the IP group was unchanged until day 4 , which was 3 days after Shigella infection induction. In the present study the dose challenge in the IP group was set as $5 \times 10^{7} \mathrm{CFU}$ in order to reduce the 
incidence of mortality; this dosage was lower than that used by both Yang et al (13) and Sharma et al (27), at 5x10 $\mathrm{CFU}$ and $10^{8}$ $\mathrm{CFU}$, respectively. In these previous studies, all mice in the IP group died within 7 days $(13,27)$. Although in the present study, the symptoms of dysentery were pronounced and persistent, a dose-dependent relationship may exist between $S$. flexneri and the diversity of the gut microbiota.

To investigate the effect of Shigella infection on the relative abundance of specific microbiome taxa, relative abundances of the species in each group on days 2, 3 and 4 were analyzed in the present study. The present results suggested that the bacteria taxa of the oral group significantly varied compared with the control and IP groups. On days 2 and 3, the relative abundances of Prevotellaceae and Alloprevotella were higher compared with the IP group and control group, while Firmicutes, Lactobacillaceae and Lactobacillus had lower abundances compared with the IP group and control group. On day 4, there was no significant difference between the oral group and the other two groups. Therefore, the present results suggested that the effect of oral infection on microbiome taxa in mice was relatively rapid, since this route of infection directly exposes the gut microbiome to S. flexneri. Probiotics such as Lactobacillus may inhibit the proliferation of Shigella, and are constantly consumed when fighting against Shigella, which results in significant decreases in their abundance (32). Several mechanisms have been suggested for the inhibitory activity of lactic acid bacteria against pathogenic bacteria, especially Gram-negative pathogens (33). These mechanisms include the production of organic acids, hydrogen peroxide and bacteriocin, as well as the competition for colonization sites with pathogenic bacteria $(32,33)$. The protective effects of Lactobacilli, probiotic bacteria, are greater against invasive bacteria such as $S$. sonnei, when compared to non-invasive bacteria such as Vibrio cholerae (34). However, the mechanism of the inhibitory effect of lactic acid bacteria on Shigella has been mostly investigated in vitro $(35,36)$, and it is not fully understood how these inhibitory effects are exerted on pathogenic bacteria in the complex intestinal microecological environment. These inhibitory effects may be associated with decreases in host gene expression, microRNA regulation and a substantial reshaping of the Listeria-monocytogenes transcriptome (37). In the present study, on days 2 and 3, the abundance of Prevotellaceae significantly increased in the oral group compared with the control group. A similar trend was also observed in the IP group, indicating that the abundance of Prevotellaceae was affected by Shigella infection. Certain Prevotella strains have been reported to serve as clinically pivotal pathobionts that participate in human diseases by promoting chronic inflammation, such as in intestinal disorders with HIV infection (38), irritable bowel syndrome (39), rheumatic arthritis (40) and periodontitis (41). Therefore, the increased abundance of Prevotella after Shigella oral infection observed in the present study likely co-induces inflammatory responses via pathogenic bacteria and recruitment of inflammatory cells. However, the specific mechanism needs to be further investigated.

The present PCoA analysis results suggested that at the initial stage of infection (day 2), the coordinates of the samples in the IP group and the oral group were divided into two distinct groups. At day 3, the distribution differences between the two groups reduced, but the gap between the two groups broadened at day 4, indicating that Shigella infection could affect the structure and composition of the intestinal flora at different time points. In addition, this effect correlated with the different symptoms and disease outcomes. For example, on the second day following oral infection of Shigella appeared to be the initial stage of shigellosis, but the symptoms had completely disappeared at day 4, while days 2-4 after IP infection indicated the progression of symptoms. The present results suggested that there were differences in shigellosis outcomes between the two infective pathways, based on the examination of intestinal flora. In addition, it has been shown that the invasion of Shigella into the large intestine via the abdominal cavity is by migration in the serosa and muscular layer, rather than via the blood circulation, suggesting that the early invasion of the pathogen from the abdominal cavity into the intestinal tract can escape the endogenous defense system of the host (13). The present results suggested that the influence of Shigella infection on intestinal flora is weak and slow at the early stage, but gradually becomes more pronounced with the development of bacteremia.

The present LEfSe analysis results suggested that Lactobacillus reuteri and Faecalitalea may be biomarkers of the control group. Previous studies have demonstrated that these bacterial groups are probiotics with certain protective effects, such as eliminating infections, attenuating both GI diseases and diseases in remote tissues, producing lactate and butyrate $(42,43)$. The direct supplementation and prebiotic modulation of Lactobacillus reuteri may be an attractive preventive and therapeutic strategy against inflammatory diseases (43). The strain Lactobacillus reuteri WHH1689 has no lactose utilization capability and exhibits a high survival rate during storage at room temperature in drinkable yogurts (44). In addition, thisstrain has shown great resistance to conditions that simulate the gastrointestinal tract, including strong adherence to HT-29 cells and inhibitory activities against Escherichia coli, S. flexneri, Salmonella paratyphi $\beta$ and Staphylococcus aureus (44). Faecalibacterium prausnitzii can inhibit the release of IL-8 and exert anti-inflammatory effects (45). Faecalibacterium prausnitzii can also inhibit the invasion of pathogenic bacteria via colonization resistance (46). Therefore, further research and clinical intervention evaluations are required to investigate the use of probiotics for the prevention and treatment of Shigella infection. One of the biomarkers in the IP group was Lachnospiraceae bacterium COE1. The members of the Lachnospiraceae family are known to influence the development of obesity and diabetes in mice with a genetic susceptibility to obesity (47). In addition, long-term high-fat feeding causes obesity-related inflammation of the ileum and colon, and increases the expression of catenin, a colon cancer risk factor, in the colon accompanied by an increase of Lachnospiraceae and Streptococcaceae abundance in the hindgut of C57BL/6 mice (48). Therefore, the increased abundance of Lachnospiraceae may affect intestinal metabolites, which reflect the intestinal metabolic response to Shigella infection.

In conclusion, the present results suggested that $S$.flexneri infection in mice can influence the profile of the gut microbiota, and the change of some specific taxa may reflect the results of Shigella-microbiota interaction, such as the decrease abundance of probiotic Lactobacillus and the increased 
abundance of Prevotellaceae. The oral and IP challenges of S. flexneri exerted different effects on the intestinal flora, including on the diversity, relative abundance and composition of the microbiota. The present results suggested that intestinal flora may serve as a barrier to Shigella transoral infection, while parenteral infection results in serious clinical manifestations due to the absence of gut microbiome inhibition. In future studies, metabonomics, metagenome and transcriptomics are required to characterize the precise mechanism of interaction between Shigella, the host and the gut microbiota.

\section{Acknowledgements}

Not applicable.

\section{Funding}

This study was financially supported by a grant from The Open Project of Key Laboratory of Environmental Factors and Cancer of Fujian Medical University (grant no. GWSZD-201801).

\section{Availability of data and materials}

The datasets used and/or analyzed during the present study are available from the corresponding author on reasonable request.

\section{Authors' contributions}

JY and WC participated in raising the animals, DNA extraction and drafting of the manuscript. PX performed the statistical analysis and bioinformatics analysis. WZ participated in the study design and revised the manuscript. All authors read and approved the final manuscript.

\section{Ethics approval and consent to participate}

The current study was approved by The Institutional Animal Care and Use Committee of Fujian Medical University. All animal care and experimental procedures were conducted according to the institutional ethical guidelines.

\section{Patient consent for publication}

Not applicable.

\section{Competing interests}

The authors declare that they have no competing interests.

\section{References}

1. Kotloff KL, Riddle MS, Platts-Mills JA, Pavlinac P and Zaidi AKM: Shigellosis. Lancet 391: 801-812, 2018.

2. Chang Z, Zhang J, Ran L, Sun J, Liu F, Luo L, Zeng L, Wang L, Li Z, Yu H and Liao Q: The changing epidemiology of bacillary dysentery and characteristics of antimicrobial resistance of Shigella isolated in China from 2004-2014. BMC Infect Dis 16: $685,2016$.

3. Taneja $\mathrm{N}$ and Mewara A: Shigellosis: Epidemiology in India. Indian J Med Res 143: 565-576, 2016.

4. Mattock E and Blocker AJ: How do the virulence factors of shigella work together to cause disease? Front Cell Infect Microbiol 7: 64, 2017.
5. Takeuchi A: Early colonic lesions in experimental Shigella infection in rhesus monkeys: Revisited. Vet Pathol Suppl 7: 1-8, 1982.

6. Kotloff KL, Winickoff JP, Ivanoff B, Clemens JD, Swerdlow DL, Sansonetti PJ, Adak GK and Levine MM: Global burden of Shigella infections: Implications for vaccine development and implementation of control strategies. Bull World Health Organ 77: 651-666, 1999.

7. Al-Soub H, Al-Maslamani M, Al-Khuwaiter J, El-Deeb Y and El-Shafie SS: Shigella flexneri perinephric abscess and bacteremia. Ann Saudi Med 25: 419-421, 2005.

8. Drow DL, Mercer L and Peacock JB: Splenic abscess caused by Shigella flexneri and Bacteroides fragilis. J Clin Microbiol 19: 79-80, 1984.

9. Amstey MS and Gandell DL: Salpingitis-perihepatitis in a patient with cervical Shigella sonnei. Obstet Gynecol 55 (3 Suppl): 70S-71S, 1980.

10. Jasper JM and Ward MA: Shigella vulvovaginitis in a prepubertal child. Pediatr Emerg Care 22: 585-586, 2006.

11. Upadhyay AK and Neely JA: Toxic megacolon and perforation caused by Shigella. Br J Surg 76: 1217, 1989.

12. Miron D, Sochotnick I, Yardeni D, Kawar B and Siplovich L: Surgical complications of shigellosis in children. Pediatr Infect Dis J 19: 898-900, 2000.

13. Yang JY, Lee SN, Chang SY, Ko HJ, Ryu S and Kweon MN: A mouse model of shigellosis by intraperitoneal infection. J Infect Dis 209: 203-215, 2014.

14. Sommer F and Backhed F: The gut microbiota-masters of host development and physiology. Nat Rev Microbiol 11: 227-238, 2013.

15. Vogt SL and Finlay BB: Gut microbiota-mediated protection against diarrheal infections. J Travel Med 24 (Suppl 1): S39-S43, 2017.

16. Bhattacharjee S, Kalbfuss N and Prazeres da Costa C: Parasites, microbiota and metabolic disease. Parasite Immunol: 39, 2017 doi: 10.1111/pim.12390.

17. Munoz-Vargas L, Opiyo SO, Digianantonio R, Williams ML, Wijeratne A and Habing G: Fecal microbiome of periparturient dairy cattle and associations with the onset of Salmonella shedding. PLoS One 13: e0196171, 2018.

18. Pearson JA, Tai N, Ekanayake-Alper DK, Peng J, Hu Y, Hager K, Compton S, Wong FS, Smith PC and Wen L: Norovirus changes susceptibility to type 1 diabetes by altering intestinal microbiota and immune cell functions. Front Immunol 10: 2654, 2019.

19. Sun X, Gao Y, Wang X, Hu G, Wang Y, Feng B, Hu Y, Mu X, Zhang Y and Dong H: Escherichia coli O101-induced diarrhea develops gut microbial dysbiosis in rats. Exp Ther Med 17: 824-834, 2019.

20. Olfert ED and Godson DL: Humane endpoints for infectious disease animal models. ILAR J 41: 99-104, 2000.

21. Edgar RC: UPARSE: Highly accurate OTU sequences from microbial amplicon reads. Nat Methods 10: 996-998, 2013.

22. Hugerth LW and Andersson AF: Analysing microbial community composition through amplicon sequencing: From sampling to hypothesis testing. Front Microbiol 8: 1561, 2017.

23. Lozupone C, Lladser ME, Knights D, Stombaugh J and Knight R: UniFrac: An effective distance metric for microbial community comparison. ISME J 5: 169-172, 2011.

24. Ramette A and Tiedje JM: Multiscale responses of microbial life to spatial distance and environmental heterogeneity in a patchy ecosystem. Proc Natl Acad Sci USA 104: 2761-2766, 2007.

25. Segata N, Izard J, Waldron L, Gevers D, Miropolsky L, Garrett WS and Huttenhower C: Metagenomic biomarker discovery and explanation. Genome Biol 12: R60, 2011.

26. Ashkenazi S: Shigella infections in children: New insights. Semin Pediatr Infect Dis 15: 246-252, 2004.

27. Sharma D, Yagnik B, Baksi R, Desai N, Padh H and Desai P: Shigellosis murine model established by intraperitoneal and intranasal route of administration: A comparative comprehension overview. Microbes Infect 19: 47-54, 2017

28. Fernandez MI, Thuizat A, Pedron T, Neutra M, Phalipon A and Sansonetti PJ: A newborn mouse model for the study of intestinal pathogenesis of shigellosis. Cell Microbiol 5: 481-491, 2003.

29. Q S Medeiros PH, Ledwaba SE, Bolick DT, Giallourou N, Yum LK, Costa DVS, Oriá RB, Barry EM, Swann JR, Lima AÂM, et al: A murine model of diarrhea, growth impairment and metabolic disturbances with Shigella flexneri infection and the role of zinc deficiency. Gut Microbes 10: 615-630, 2019.

30. Kim BR, Shin J, Guevarra R, Lee JH, Kim DW, Seol KH, Lee JH, Kim HB and Isaacson R: Deciphering diversity indices for a better understanding of microbial communities. J Microbiol Biotechnol 27: 2089-2093, 2017. 
31. Shim DH, Ryu S and Kweon MN: Defensins play a crucial role in protecting mice against oral Shigella flexneri infection. Biochem Biophys Res Commun 401: 554-560, 2010.

32. Davoodabadi A, Soltan Dallal MM, Lashani E and Tajabadi Ebrahimi M: Antimicrobial Activity of Lactobacillus spp. Isolated from fecal flora of healthy breast-fed infants against diarrheagenic Escherichia coli. Jundishapur J Microbiol 8: e27852, 2015.

33. Zhang YC, Zhang LW, Ma W, Yi HX, Yang X, Du M, Shan YJ, Han $X$ and Zhang LL: Screening of probiotic lactobacilli for inhibition of Shigella sonnei and the macromolecules involved in inhibition. Anaerobe 18: 498-503, 2012.

34. Alamdary SZ, Bakhshi B and Soudi S: The anti-apoptotic and anti-inflammatory effect of Lactobacillus acidophilus on Shigella sonnei and Vibrio cholerae interaction with intestinal epithelial cells: A comparison between invasive and non-invasive bacteria. PLoS One 13: e0196941, 2018.

35. Mirnejad R, Vahdati AR, Rashidiani J, Erfani M and Piranfar V: The antimicrobial effect of lactobacillus casei culture supernatant against multiple drug resistant clinical isolates of Shigella sonnei and Shigella flexneri in vitro. Iran Red Crescent Med J 15: 122-126, 2013

36. Zhang Y, Shi X, Hao S, Lu Q, Zhang L, Han X and Lu W: Inhibition of Shigella sonnei-induced epithelial barrier disruption by surface-layer associated proteins of lactobacilli from Chinese fermented food. J Dairy Sci 101: 1834-1842, 2018.

37. Archambaud C, Nahori MA, Soubigou G, Bécavin C, Laval L, Lechat P, Smokvina T, Langella P, Lecuit M and Cossart P: Impact of lactobacilli on orally acquired listeriosis. Proc Natl Acad Sci USA 109: 16684-16689, 2012.

38. Larsen JM: The immune response to Prevotella bacteria in chronic inflammatory disease. Immunology 151: 363-374, 2017.

39. Su T, Liu R, Lee A, Long Y, Du L, Lai S, Chen X, Wang L, Si J, Owyang C and Chen S: Altered intestinal microbiota with increased abundance of Prevotella is associated with high risk of diarrhea-predominant irritable bowel syndrome. Gastroenterol Res Pract 2018: 6961783, 2018.

40. Maeda Y and Takeda K: Role of gut microbiota in rheumatoid arthritis. J Clin Med 6: pii: E60, 2017.
41. Szafranski SP, Deng ZL, Tomasch J, Jarek M, Bhuju S, Meisinger C, Kühnisch J, Sztajer H and Wagner-Döbler I: Functional biomarkers for chronic periodontitis and insights into the roles of Prevotella nigrescens and Fusobacterium nucleatum; a metatranscriptome analysis. NPJ Biofilms Microbiomes 1: 15017, 2015

42. Kageyama A, Benno Y and Nakase T: Phylogenetic and phenotypic evidence for the transfer of Eubacterium aerofaciens to the genus Collinsella as Collinsella aerofaciens gen. nov., comb. nov. Int J Syst Bacteriol 49: 557-565, 1999.

43. Mu Q, Tavella VJ and Luo XM: Role of Lactobacillus reuteri in human health and diseases. Front Microbiol 9: 757, 2018.

44. Chen S, Chen L, Chen L, Ren X, Ge H, Li B, Ma G, Ke X, Zhu J, Li L, et al: Potential probiotic characterization of Lactobacillus reuteri from traditional Chinese highland barley wine and application for room-temperature-storage drinkable yogurt. J Dairy Sci 101: 5780-5788, 2018

45. Sokol H, Pigneur B, Watterlot L, Lakhdari O, Bermúdez-Humarán LG, Gratadoux JJ, Blugeon S, Bridonneau C, Furet JP, Corthier G, et al: Faecalibacterium prausnitzii is an anti-inflammatory commensal bacterium identified by gut microbiota analysis of Crohn disease patients. Proc Natl Acad Sci USA 105: 16731-16736, 2008.

46. Benus RF, Harmsen HJ, Welling GW, Spanjersberg R, Zijlstra JG, Degener JE and van der Werf TS: Impact of digestive and oropharyngeal decontamination on the intestinal microbiota in ICU patients. Intensive Care Med 36: 1394-1402, 2010.

47. Kameyama $\mathrm{K}$ and Itoh $\mathrm{K}$ : Intestinal colonization by a Lachnospiraceae bacterium contributes to the development of diabetes in obese mice. Microbes Environ 29: 427-430, 2014.

48. Zeng H, Ishaq SL, Zhao FQ and Wright AG: Colonic inflammation accompanies an increase of $\beta$-catenin signaling and Lachnospiraceae/Streptococcaceae bacteria in the hind gut of high-fat diet-fed mice. J Nutr Biochem 35: 30-36, 2016. International (CC BY-NC-ND 4.0) License. 